\title{
Symmetry properties and explicit solutions of the nonlinear time fractional KdV equation
}

\author{
Gangwei Wang ${ }^{*}$ and Tianzhou Xu
}

"Correspondence: pukai1121@163.com

School of Mathematics, Beijing Institute of Technology, Beijing, 100081, P.R. China

\begin{abstract}
The time fractional KdV equation in the sense of the Riemann-Liouville derivatives is considered. The symmetry properties of the time fractional $\mathrm{KdV}$ equation is investigated by using the Lie group analysis method. On the basis of the point symmetry, the vector fields of the time fractional KdV equation are presented. And then, the symmetry reductions are constructed. By right of the obtained Lie point symmetries, it is shown that this equation could transform into a nonlinear ordinary differential equation of fractional order with the new independent variable $\xi=x t^{-\alpha / 3}$. The derivative is an Erdélyi-Kober derivative depending on a parameter $\alpha$. At last, by means of the sub-equation method, some exact and explicit solutions of the time fractional KdV equation are constructed.

MSC: Primary 22E70; secondary 26A33
\end{abstract}

Keywords: fractional KdV equation; modified Riemann-Liouville derivative; Lie symmetry analysis; Erdélyi-Kober operators; exact solutions

\section{Introduction}

Lie point transformation groups have been extensively applied to the nonlinear partial differential equations arising in mathematics, physics and in many other scientific fields. Lie point transformation groups can deal with symmetry reductions, similarity solutions of nonlinear differential partial equations. It can be said that the method of Lie symmetry groups is the most important approach for constructing analytical solutions of nonlinear partial differential equations. A huge number of papers and many excellent textbooks (see, e.g., [1-16] and papers cited therein) have been devoted to the theory of Lie symmetry groups and their applications to differential equations. On the other hand, it is important to note that a very small number of them involve Lie group analysis to solve problems for fractional differential equations (FDEs) [17-23]. As is known to all, there is no existing general method for dealing with fractional differential equations. Lie group analysis, however, is a powerful, systematic and direct method for investigating fractional differential equations. It is a fact that the classical Lie theory applied to fractional differential equations (FDEs) for symmetry analysis should be modified since it fails in the case of fractional differential equations.

Since Leibniz's note in his letter to L'Hospital, in which the meaning of the derivative of order one half was firstly discussed, the theory of derivatives of non-integer order has stimulated considerable interest in the areas of mathematics, physics and engineering [2428]. Recently, the fractional calculus has been used successfully to describe many complex 
nonlinear phenomena and dynamic processes in physics, engineering, electromagnetics, acoustics, viscoelasticity, electrochemistry, material science, etc. [24-40]. In reality, the next state of a physical phenomenon might depend not only on its current state but also on its historical states (non-local property), which can be successfully modeled by using the theory of derivatives and integrals of fractional order [21, 22]. Because of this, many effective analytic methods have been developed to derive exact, explicit and numerical solutions of nonlinear fractional partial differential equations (FPDEs), such as the Adomian decomposition method [29,30], the invariant subspace method [31], the transform method [32, 33], the homotopy perturbation method [34], the variational iteration method [35], the sub-equation method [36-38], and so on.

In this paper, by means of the Lie symmetry group method, we consider the following nonlinear fractional $\mathrm{KdV}(\mathrm{FK})$ equation with time fractional derivatives of the form

$$
\frac{\partial^{\alpha} u}{\partial t^{\alpha}}+a u u_{x}+b u_{x x x}=0
$$

where $0<\alpha \leq 1, a, c$ are parameters, $\alpha$ is a parameter describing the order of the fractional time derivative. The function $u(x, t)$ is assumed to be a causal function of time. In physical applications, the special cases such as $\alpha=\frac{1}{2}$ and $\alpha=\frac{1}{3}$ may be more useful. In the case of $\alpha=1$, the fractional equation reduces to the classical nonlinear KdV equation. In [21], invariant analysis of the time fractional generalized Korteweg-de Vries equation is investigated. However, the authors do not give explicit solutions.

The paper is organized as follows. In Section 2, a brief review of the main definitions and properties of fractional calculus and general concept of the invariance criterion for FDEs are given to provide a convenient reference. In Section 3, we perform Lie group classification on the time fractional KdV equation, the general similarity forms and symmetry reductions are established. In Section 4, some exact solutions are discussed. The conclusions are presented in Section 5.

\section{Preliminaries}

We state some notations and theorems. First, let us recall essentials of fractional calculus. The fractional calculus is a name for the theory of integrals and derivatives of arbitrary order. It unifies and generalizes the notions of integer-order differentiation and $n$-fold integration. Besides the R-L definition of fractional derivatives, there are several other different definitions, such as the modified R-L (mR-L) derivative, the Grünwald-Letnikov derivative (G-L) and Caputo's fractional derivative, and so on. For different circumstances, they can be used for dealing with different properties of physical models. For example, Caputo's fractional derivative is related to initial value problems; generally speaking, the $\mathrm{mR}-\mathrm{L}$ derivative is used to investigate exact and explicit solutions of some FDEs [36-38]. The modified Riemann-Liouville derivative was defined by Jumarie [39, 40].

\section{Definition 2.1}

$$
D_{t}^{\alpha} f(t)= \begin{cases}\frac{1}{\Gamma(1-\alpha)} \frac{d}{d t} \int_{0}^{t}(t-\xi)^{-\alpha}(f(\xi)-f(0)) d \xi, & 0<\alpha<1 \\ {\left[f^{(n)}(t)\right]^{(\alpha-n)},} & n \leq \alpha<n+1, n \geq 1\end{cases}
$$


where the Euler gamma function $\Gamma(z)$ is defined by the integral

$$
\Gamma(z)=\int_{0}^{\infty} e^{-t} t^{z-1} d t
$$

which converges in the right half of the complex plane $\operatorname{Re}(z)>0$.

\section{Properties 2.1}

$$
\begin{aligned}
& D_{t}^{\alpha} t^{\gamma}=\frac{\Gamma(\gamma+1)}{\Gamma(\gamma+1-\alpha)} t^{\gamma-\alpha}, \quad \gamma>0, \\
& D_{t}^{\alpha}[u(t) v(t)]=u(t) D_{t}^{\alpha} v(t)+v(t) D_{t}^{\alpha} u(t), \\
& D_{t}^{\alpha}[f(u(t))]=f_{u}^{\prime}[u(t)] D_{t}^{\alpha} u(t)=D_{u}^{\alpha} f[u(t)]\left(u_{t}^{\prime}\right)^{\alpha},
\end{aligned}
$$

which will be used in the following sections.

In what follows, considering the general case of a nonlinear system of partial differential equations of order $n$ in $p$ independent and $q$ dependent variables $[1,2,4-7]$

$$
\Delta_{v}\left(x, u^{(n)}\right)=0, \quad v=1,2,3, \ldots, l,
$$

here $x=\left(x^{1}, x^{2}, \ldots, x^{p}\right), u=\left(u^{1}, u^{2}, \ldots, u^{q}\right)$, and the derivatives of $u$ in reference to $x$ up to $n$, where $u^{(n)}$ represents all the derivatives of $u$ of all orders from 0 to $n$. Consider a one-parameter Lie group of infinitesimal transformations acting on system (7),

$$
\begin{aligned}
& \bar{x}^{i}=x^{i}+\epsilon \xi^{i}(x, u)+O\left(\epsilon^{2}\right), \quad i=1, \ldots, p, \\
& \bar{u}^{j}=u^{j}+\epsilon \eta^{j}(x, u)+O\left(\epsilon^{2}\right), \quad j=1, \ldots, q,
\end{aligned}
$$

where $\epsilon$ is the parameter. The vector field $V$ is associated with the above group of transformations as follows:

$$
V=\sum_{i=1}^{p} \xi^{i}(x, u) \partial_{x^{i}}+\sum_{\alpha=1}^{q} \eta^{\alpha}(x, u) \partial_{u}^{\alpha}
$$

The invariance of system (7) under the infinitesimal transformations leads to the invariance conditions

$$
\operatorname{pr}^{(n)} V\left[\Delta_{v}\left(x, u^{(n)}\right)\right]=0, \quad v=1,2,3, \ldots, l
$$

whenever $\Delta_{v}\left(x, u^{(n)}\right)=0$, where $\mathrm{pr}^{(n)} V$ is called the $n$th order prolongation of the infinitesimal generator given by

$$
\operatorname{pr}^{(n)} V=V+\sum_{\alpha=1}^{q} \sum_{J} \eta_{\alpha}^{J}\left(x, u^{(n)}\right) \partial_{u_{j}^{\alpha}},
$$


where $J=\left(j_{1}, j_{2}, \ldots, j_{k}\right)$, with $1 \leq j_{k} \leq p, 1 \leq k \leq n$. The coefficient functions $\eta_{\alpha}^{J}$ of $\operatorname{pr}^{(n)} V$ are given by the following formula:

$$
\eta_{\alpha}^{J}\left(x, u^{(n)}\right)=D_{J}\left(\eta_{\alpha}-\sum_{i=1}^{p} \xi^{i} u_{i}^{\alpha}\right)+\sum_{i=1}^{p} \xi^{i} u_{J, i}^{\alpha},
$$

where $u_{i}^{\alpha}=\partial u^{\alpha} / \partial x^{i}$ and $u_{J, i}^{\alpha}=\partial u_{J}^{\alpha} / \partial x^{i}$.

However, for a nonlinear system of fractional partial differential equations (FPDEs), the prolongation formula will be different. Now, we present below brief details of the Lie symmetry analysis to FPDEs with respect to two independent variables.

Consider a scalar time FPDE having the following form [21, 22]:

$$
\frac{\partial^{\alpha} u}{\partial t^{\alpha}}=F\left(x, t, u, u_{x}, u_{x x}, \ldots\right)
$$

If (13) is invariant under a one-parameter Lie group of point transformations

$$
\begin{aligned}
& t^{*}=t+\epsilon \tau(x, t, u)+O\left(\epsilon^{2}\right), \\
& x^{*}=x+\epsilon \xi(x, t, u)+O\left(\epsilon^{2}\right), \\
& u^{*}=u+\epsilon \eta(x, t, u)+O\left(\epsilon^{2}\right), \\
& \frac{\partial^{\alpha} \bar{u}}{\partial \bar{t}^{\alpha}}=\frac{\partial^{\alpha} u}{\partial t^{\alpha}}+\epsilon \eta_{\alpha}^{0}(x, t, u)+O\left(\epsilon^{2}\right), \\
& \frac{\partial \bar{u}}{\partial \bar{x}}=\frac{\partial u}{\partial x}+\epsilon \eta^{x}(x, t, u)+O\left(\epsilon^{2}\right), \\
& \frac{\partial^{2} \bar{u}}{\partial \bar{x}^{2}}=\frac{\partial^{2} u}{\partial x^{2}}+\epsilon \eta^{x x}(x, t, u)+O\left(\epsilon^{2}\right),
\end{aligned}
$$

where

$$
\begin{aligned}
\eta_{\alpha}^{0}= & D_{t}^{\alpha}(\eta)+\xi D_{t}^{\alpha}\left(u_{x}\right)-D_{t}^{\alpha}\left(\xi u_{x}\right)+D_{t}^{\alpha}\left(D_{t}(\tau) u\right)-D_{t}^{\alpha+1}(\tau u)+\tau D_{t}^{\alpha+1}(u) \\
= & \frac{\partial^{\alpha} \eta}{\partial t^{\alpha}}+\left(\eta_{u}-\alpha D_{t}(\tau)\right) \frac{\partial^{\alpha} u}{\partial t^{\alpha}}-u \frac{\partial^{\alpha} \eta_{u}}{\partial t^{\alpha}}+\mu \\
& +\sum_{n=1}^{\infty}\left[\left(\begin{array}{l}
a \\
n
\end{array}\right) \frac{\partial^{\alpha} \eta_{u}}{\partial t^{\alpha}}-\left(\begin{array}{c}
a \\
n+1
\end{array}\right) D_{t}^{n+1}(\tau)\right] D_{t}^{\alpha-n}(u) \\
& -\sum_{n=1}^{\infty}\left(\begin{array}{l}
a \\
n
\end{array}\right) D_{t}^{n}(\xi) D_{t}^{\alpha-n}\left(u_{x}\right)
\end{aligned}
$$

where

$$
\mu=\sum_{n=2}^{\infty} \sum_{m=2}^{n} \sum_{k=2}^{m} \sum_{r=0}^{k-1}\left(\begin{array}{l}
a \\
n
\end{array}\right)\left(\begin{array}{l}
n \\
m
\end{array}\right)\left(\begin{array}{l}
k \\
r
\end{array}\right) \frac{1}{k !} \frac{t^{n-\alpha}}{\Gamma(n+1-\alpha)}[-u]^{r} \frac{\partial^{m}}{\partial t^{m}}\left[u^{k-r}\right] \frac{\partial^{n-m+k} \eta}{\partial t^{n-m} \partial u^{k}}
$$


and

$$
\begin{aligned}
& \eta^{x}=D_{x}(\eta)-u_{x} D_{x}(\xi)-u_{t} D_{x}(\tau), \\
& \eta^{x x}=D_{x}\left(\eta^{x}\right)-u_{x t} D_{x}(\tau)-u_{x x} D_{x}(\xi), \\
& \eta^{x x x}=D_{x}\left(\eta^{x x}\right)-u_{x x t} D_{x}(\tau)-u_{x x x} D_{x}(\xi),
\end{aligned}
$$

Here, $D_{x}$ denotes the total derivative operator and it is defined by

$$
D_{x}=\frac{\partial}{\partial x}+u_{x} \frac{\partial}{\partial u}+u_{x x} \frac{\partial}{\partial u_{x}}+\cdots
$$

with the associated vector field of the form

$$
V=\tau(x, t, u) \frac{\partial}{\partial t}+\xi(x, t, u) \frac{\partial}{\partial x}+\eta(x, t, u) \frac{\partial}{\partial u},
$$

where the coefficient functions $\xi(x, t, u), \tau(x, t, u)$, and $\eta(x, t, u)$ of the vector field are to be determined.

If vector field (19) generates a symmetry of (1), then $V$ must satisfy Lie's symmetry condition

$$
\left.\operatorname{pr}^{(n)} V\left(\Delta_{1}\right)\right|_{\Delta_{1}=0}=0
$$

where $\Delta_{1}=\frac{\partial^{\alpha} u}{\partial t^{\alpha}}-F\left(x, t, u, u_{x}, u_{x x}, \ldots\right)$.

\section{Symmetry group analysis of the nonlinear time fractional $\mathrm{KdV}$ equation}

In the preceding section, we have given some definitions and formulas about the Lie symmetry analysis method on the FPDEs. In the present section, through the above discussion and the Lie theory, we investigate the time-fractional KdV equation.

According to the Lie theory, applying the third prolongation $\mathrm{pr}^{(3)} V$ to (1), we can get the following system of symmetry equations:

$$
\eta_{\alpha}^{0}+a u \eta^{x}+a \eta u_{x}+b \eta^{x x x}=0
$$

Substituting (14), (15) and (17) into (21), and equating the coefficients of the various monomials in partial derivatives with respect to $x$ and various power of $u$, one can find the determining equations for the symmetry group of Eq. (1). Solving these equations, we obtain the following forms of the coefficient functions:

$$
\xi=c_{1} x+c_{2}, \quad \tau=\frac{3 c_{1} t}{\alpha}, \quad \eta=-2 c_{1} u
$$

where $c_{1}$ and $c_{2}$ are arbitrary constants. Therefore, we can obtain the corresponding vector fields

$$
V=\frac{3 c_{1} t}{\alpha} \frac{\partial}{\partial t}+\left(c_{1} x+c_{2}\right) \frac{\partial}{\partial x}-2 c_{1} u \frac{\partial}{\partial u}
$$


Thus, the Lie point symmetries are represented by the Lie group generators (vector fields)

$$
V_{1}=\frac{\partial}{\partial x}, \quad V_{2}=\frac{3 t}{\alpha} \frac{\partial}{\partial t}+x \frac{\partial}{\partial x}-2 u \frac{\partial}{\partial u} .
$$

Moreover, it is easily checked that the vector fields are closed under the Lie bracket, respectively,

$$
\left[V_{1}, V_{2}\right]=V_{1}, \quad\left[V_{2}, V_{1}\right]=-V_{1} .
$$

For the symmetry $V_{2}$, we get the characteristic equation

$$
\frac{d x}{x}=\frac{\alpha d t}{3 t}=\frac{d u}{-2 u}
$$

and the corresponding invariants are

$$
\xi=x t^{\frac{-\alpha}{3}}, \quad u=t^{\frac{-2 \alpha}{3}} g(\xi) .
$$

From what has been discussed above, one can see that (1) can be reduced to a nonlinear ODE of fractional order with a new independent variable $\xi=x t^{-\alpha / 3}$. Consequently, one can get the following theorem.

Theorem Transformation (27) reduces (1) to the following nonlinear ordinary differential equation of fractional order:

$$
\left(P_{\frac{3}{\alpha}}^{1-\frac{5 \alpha}{3}, \alpha} g\right)(\xi)+a g g_{\xi}+b g_{\xi \xi \xi}=0
$$

with the Erdélyi-Kober fractional differential operator $P_{\beta}^{\tau, \alpha}$ of order [28]

$$
\begin{aligned}
& \left(P_{\beta}^{\tau, \alpha} g\right):=\prod_{j=0}^{n-1}\left(\tau+j-\frac{1}{\beta} \xi \frac{d}{d \xi}\right)\left(K_{\beta}^{\tau+\alpha, n-\alpha} g\right)(\xi), \\
& n= \begin{cases}{[\alpha]+1,} & \alpha \notin \mathrm{N}, \\
\alpha, & \alpha \in \mathrm{N},\end{cases}
\end{aligned}
$$

where

$$
\left(K_{\beta}^{\tau, \alpha} g\right)(\xi):= \begin{cases}\frac{1}{\Gamma(\alpha)} \int_{1}^{\infty}(u-1)^{\alpha-1} u^{-(\tau+\alpha)} g\left(\xi u^{\frac{1}{\beta}}\right) d u, & \alpha>0, \\ g(\xi), & \alpha=0\end{cases}
$$

is the Erdélyi-Kober fractional integral operator.

Proof Let $n-1<\alpha<n, n=1,2,3, \ldots$. Considering the Riemann-Liouville fractional derivative, one can get

$$
\frac{\partial^{\alpha} u}{\partial t^{\alpha}}=\frac{\partial^{n}}{\partial t^{n}}\left[\frac{1}{\Gamma(n-\alpha)} \int_{0}^{t}(t-s)^{n-\alpha-1} s^{\frac{-\alpha}{3}} g\left(x s^{\frac{-\alpha}{3}}\right) d s\right] .
$$


Let $v=\frac{t}{s}$, one can get $d s=-\frac{t}{v^{2}} d v$, thus (32) can write

$$
\frac{\partial^{\alpha} u}{\partial t^{\alpha}}=\frac{\partial^{n}}{\partial t^{n}}\left[t^{n-\frac{5 \alpha}{3}} \frac{1}{\Gamma(n-\alpha)} \int_{1}^{\infty}(v-1)^{n-\alpha-1} v^{-\left(n+1-\frac{5 \alpha}{3}\right)} g\left(\xi v^{\frac{\alpha}{3}}\right) d v\right]
$$

Based on the Erdélyi-Kober fractional integral operator (31), one can have

$$
\frac{\partial^{\alpha} u}{\partial t^{\alpha}}=\frac{\partial^{n}}{\partial t^{n}}\left[t^{n-\frac{5 \alpha}{3}}\left(K_{\frac{\alpha}{3}}^{1-\frac{2 \alpha}{3}, n-\alpha} g\right)(\xi)\right]
$$

In view of the relation $\left(\xi=x t^{\frac{-\alpha}{3}}\right)$, we can get

$$
t \frac{\partial}{\partial t} \phi(\xi)=t x\left(-\frac{\alpha}{3}\right) t^{-\frac{\alpha}{3}-1} \phi^{\prime}(\xi)=-\frac{\alpha}{3} \xi \frac{\partial}{\partial \xi} \phi(\xi) .
$$

Thus, one can obtain

$$
\begin{aligned}
\frac{\partial^{n}}{\partial t^{n}}\left[t^{n-\frac{5 \alpha}{3}}\left(K_{\frac{3}{\alpha}}^{1-\frac{2 \alpha}{3}, n-\alpha} g\right)(\xi)\right] & =\frac{\partial^{n-1}}{\partial t^{n-1}}\left[\frac{\partial}{\partial t}\left(t^{n-\frac{5 \alpha}{3}}\left(K_{\frac{3}{\alpha}}^{1-\frac{2 \alpha}{3}, n-\alpha} g\right)(\xi)\right)\right] \\
& =\frac{\partial^{n-1}}{\partial t^{n-1}}\left[t^{n-\frac{5 \alpha}{3}}\left(n-\frac{5 \alpha}{3}-\frac{\alpha}{3} \xi \frac{\partial}{\partial \xi}\left(K_{\frac{3}{\alpha}}^{1-\frac{2 \alpha}{3}, n-\alpha} g\right)(\xi)\right)\right] .
\end{aligned}
$$

Repeating the similar procedure as above for $n-1$ times, one can get

$$
\begin{aligned}
& \frac{\partial^{n}}{\partial t^{n}}\left[t^{n-\frac{5 \alpha}{3}}\left(K_{\frac{3}{\alpha}}^{1-\frac{2 \alpha}{3}, n-\alpha} g\right)(\xi)\right] \\
& \quad=\frac{\partial^{n-1}}{\partial t^{n-1}}\left[\frac{\partial}{\partial t}\left(t^{n-\frac{5 \alpha}{3}}\left(K_{\frac{3}{\alpha}}^{1-\frac{2 \alpha}{3}, n-\alpha} g\right)(\xi)\right)\right] \\
& \quad=\frac{\partial^{n-1}}{\partial t^{n-1}}\left[t^{n-\frac{5 \alpha}{3}}\left(n-\frac{5 \alpha}{3}-\frac{\alpha}{3} \xi \frac{\partial}{\partial \xi}\left(K_{\frac{3}{\alpha}}^{1-\frac{2 \alpha}{3}, n-\alpha} g\right)(\xi)\right)\right] \\
& =\cdots=t^{-\frac{5 \alpha}{3}} \prod_{j=0}^{n-1}\left(1-\frac{5 \alpha}{3}+j-\frac{\alpha}{3} \xi \frac{d}{d \xi}\right)\left(K_{\frac{3}{\alpha}}^{1-\frac{2 \alpha}{3}, n-\alpha} g\right)(\xi) .
\end{aligned}
$$

Now using (29), we get

$$
\frac{\partial^{n}}{\partial t^{n}}\left[t^{n-\frac{5 \alpha}{3}}\left(K_{\frac{3}{\alpha}}^{1-\frac{2 \alpha}{3}, n-\alpha} g\right)(\xi)\right]=t^{-\frac{5 \alpha}{3}}\left(P_{\frac{3}{\alpha}}^{1-\frac{5 \alpha}{3}, n-\alpha} g\right)(\xi)
$$

Substituting (38) into (34), one can get

$$
\frac{\partial^{\alpha} u}{\partial t^{\alpha}}=t^{-\frac{5 \alpha}{3}}\left(P_{\frac{3}{\alpha}}^{1-\frac{5 \alpha}{3}, n-\alpha} g\right)(\xi)
$$

Therefore, the time fractional $\mathrm{KdV}$ equation can be reduced into an FODE (fractional ordinary differential equation)

$$
\left(P_{\frac{3}{\alpha}}^{1-\frac{5 \alpha}{3}, \alpha} g\right)(\xi)+a g g_{\xi}+b g_{\xi \xi \xi}=0
$$

This completes the proof. 
In particular, if $\alpha=\frac{1}{2}$, then the symmetries of the time fractional Korteweg-de Vries equation are as follows:

$$
V=6 c_{1} t \frac{\partial}{\partial t}+\left(c_{1} x+c_{2}\right) \frac{\partial}{\partial x}-2 c_{1} u \frac{\partial}{\partial u} .
$$

In this case, the time fractional KdV equation can be reduced into an FODE

$$
\left(P_{6}^{\frac{1}{6}, \frac{1}{2}} g\right)(\xi)+a g g_{\xi}+b g_{\xi \xi \xi}=0
$$

Similarly, in view of (23), the symmetries can be obtained successively in terms of the specific parameters $\alpha$, such as $\alpha=\frac{1}{3}, \alpha=\frac{1}{4}$ and so on.

Remark 1 Through the above discussion, we can find that the point symmetries of the time fractional KdV equation are relatively fewer than those for the generalized KdV equation. The main reason is that the fractional order $0<\alpha \leq 1$ is an arbitrary parameter in our discussion. It is appropriate to mention here that the fractional order $0<\alpha \leq 1$ affects the properties of the equation greatly.

\section{Exact and explicit solutions of the time fractional $\mathrm{KdV}$ equation}

\subsection{Fractional sub-equation method}

Now, we outline the main steps of the fractional sub-equation method for solving fractional differential equations.

For a given NFDE, consider two variables $x$ and $t$,

$$
P\left(u, u_{t}, u_{x}, D_{t}^{\alpha} u, D_{x}^{\alpha} u, \ldots\right)=0, \quad 0<\alpha \leq 1
$$

where $D_{x}^{\alpha} u$ and $D_{t}^{\alpha} u$ are the modified Riemann-Liouville derivatives of $u$ with respect to $t$ and $x$, respectively.

Step 1: By making use of the traveling wave transformation

$$
u(x, t)=u(\xi), \quad \xi=x+c t
$$

where $c$ is a nonzero constant to be determined later, (41) can be reduced to a nonlinear fractional ordinary differential equation (NFODE)

$$
P\left(u, c u^{\prime}, u^{\prime}, c^{\alpha} D_{\xi}^{\alpha} u, D_{\xi}^{\alpha} u, \ldots\right)=0, \quad 0<\alpha \leq 1
$$

Step 2: Suppose that Eq. (43) has the following solution:

$$
u(\xi)=a_{0}+\sum_{i=1}^{n} a_{i}(\psi(\xi))^{i}
$$

where $a_{i}(i=1, \ldots, n)$ are constants to be determined later, positive integer $n$ can be determined by balancing the highest order derivatives and nonlinear terms in Eq. (41) or Eq. 
(43). The function $\psi(\xi)$ satisfies the following Bäklund transformation of the fractional Riccati equation [38]:

$$
\psi(\xi)=\frac{-\sigma B+D \phi(\xi)}{D+B \phi(\xi)}
$$

where $B, D$ are arbitrary parameters, and $B \neq 0$. Meanwhile, $\phi(\xi)$ are decided by

$$
D_{\xi}^{\alpha} \phi(\xi)=\sigma+\phi^{2}(\xi)
$$

where $\sigma$ is a constant. Eq. (46) has the following solutions:

$$
\phi(\xi)= \begin{cases}-\sqrt{-\sigma} \tanh (\sqrt{-\sigma} \xi, \alpha), & \sigma<0, \\ -\sqrt{-\sigma} \operatorname{coth}(\sqrt{-\sigma} \xi, \alpha), & \sigma<0, \\ \sqrt{\sigma} \tan (\sqrt{\sigma} \xi, \alpha), & \sigma>0, \\ -\sqrt{\sigma} \cot (\sqrt{\sigma} \xi, \alpha), & \sigma>0, \\ -\frac{\Gamma(1+\alpha)}{\xi^{\alpha}+\omega}, & \omega \text { is constant, } \sigma=0,\end{cases}
$$

with the generalized hyperbolic and trigonometric functions

$$
\begin{aligned}
& \sin _{\alpha}(\xi)=\frac{E_{\alpha}\left(i \xi^{\alpha}\right)-E_{\alpha}\left(-i \xi^{\alpha}\right)}{2 i}, \quad \cos _{\alpha}(\xi)=\frac{E_{\alpha}\left(i \xi^{\alpha}\right)+E_{\alpha}\left(-i \xi^{\alpha}\right)}{2 i}, \\
& \sinh _{\alpha}(\xi)=\frac{E_{\alpha}\left(\xi^{\alpha}\right)-E_{\alpha}\left(-\xi^{\alpha}\right)}{2}, \quad \cosh _{\alpha}(\xi)=\frac{E_{\alpha}\left(\xi^{\alpha}\right)+E_{\alpha}\left(-\xi^{\alpha}\right)}{2} \\
& \tan _{\alpha}(\xi)=\frac{\sin _{\alpha}(\xi)}{\cos _{\alpha}(\xi)}, \quad \cot _{\alpha}(\xi)=\frac{\cos _{\alpha}(\xi)}{\sin _{\alpha}(\xi)} \\
& \tanh _{\alpha}(\xi)=\frac{\sinh _{\alpha}(\xi)}{\cosh _{\alpha}(\xi)}, \quad \operatorname{coth}_{\alpha}(\xi)=\frac{\cosh _{\alpha}(\xi)}{\sinh _{\alpha}(\xi)}
\end{aligned}
$$

here $E_{\alpha}(\xi)=\sum_{k=0}^{\infty} \frac{\xi^{k}}{\Gamma(1+k \alpha)}(\alpha>0)$ is the Mittag-Leffler function in one parameter.

Step 3: Substituting (44), (45) and (46) into (43) and setting the coefficients of the powers of $(\phi(\xi))^{i}$ to be zero, one can obtain an over-determined nonlinear algebraic system in $a_{i}$ $(i=1, \ldots, n)$ and $c$.

Step 4: With the aid of Maple, solving the nonlinear algebraic system yields the explicit expressions of the parameters $a_{i}(i=1, \ldots, n)$ and $c$. Then substituting these constants and the solutions of Eq. (47) into Eq. (44), we can get the exact and explicit solutions of the nonlinear fractional partial differential equation (NFPDE) (41).

\subsection{Applications to the time fractional KdV equation}

According to the above steps, firstly, we introduce the following transformations:

$$
u(x, t)=u(\xi), \quad \xi=x+c t,
$$

where $c$ is a constant. Substituting (49) into (1), then (1) can be reduced to the following nonlinear fractional ordinary differential equation (NFODE):

$$
c^{\alpha} D_{\xi}^{\alpha} u+a u u_{\xi}+b u_{\xi \xi \xi}=0 .
$$


We suppose that Eq. (50) has the following solution:

$$
u(\xi)=a_{0}+\sum_{i=1}^{n} a_{i}(\psi(\xi))^{i},
$$

where $a_{i}(i=1, \ldots, n)$ are constants to be determined later. Balancing the highest order derivative terms with nonlinear terms in Eq. (50), we get

$$
u(\xi)=a_{0}+a_{1}(\psi)+a_{2}(\psi)^{2} .
$$

Substituting (52) along with (45) into (50) and then letting the coefficients of $(\phi)^{i}$ to zero, one can get some algebraic equations about $c, a_{0}, a_{1}$ and $a_{2}$. Solving the algebraic equations by Maple, one can get the following.

Case 1:

$$
\begin{array}{ll}
B=B, & D=D, \quad a=a, \quad b=b, \quad c=\left(-a a_{0}\right)^{\frac{1}{\alpha},} \\
\alpha=\alpha, & \sigma=0, \quad a_{0}=a_{0}, \quad a_{1}=0, \quad a_{2}=-12 \frac{b}{a} .
\end{array}
$$

Case 2:

$$
\begin{aligned}
& B=B, \quad D=D, \quad a=a, \quad b=b, \quad c=c, \quad \alpha=\alpha, \\
& \sigma=-\frac{D^{2}}{B^{2}}, \quad a_{0}=a_{0}, \quad a_{1}=a_{1}, \quad a_{2}=a_{2} .
\end{aligned}
$$

Case 3:

$$
\begin{aligned}
& B=B, \quad D=D, \quad a=-12 \frac{b}{a_{2}}, \quad b=b, \quad c=\left(-4 \frac{b\left(2 a_{2} \sigma-3 a_{0}\right)}{a_{2}}\right)^{\frac{1}{\alpha}}, \\
& \alpha=\alpha, \quad \sigma=\sigma, \quad a_{0}=a_{0}, \quad a_{1}=0, \quad a_{2}=a_{2} .
\end{aligned}
$$

Case 4:

$$
\begin{aligned}
& B=B, \quad D=0, \quad a=a, \quad b=-\frac{1}{12} a a_{2}, \quad c=\left(\frac{2}{3} a \sigma a_{2}-a a_{0}\right)^{\frac{1}{\alpha}}, \\
& \alpha=\alpha, \quad \sigma=\sigma, \quad a_{0}=a_{0}, \quad a_{1}=0, \quad a_{2}=a_{2} .
\end{aligned}
$$

In view of (54), one can get new types of explicit solutions of Eq. (1) as follows:

$$
\begin{aligned}
u= & a_{0}+a_{1}\left(\frac{-\sigma B-D \sqrt{-\sigma} \tanh (\sqrt{-\sigma} \xi, \alpha)}{D-B \sqrt{-\sigma} \tanh (\sqrt{-\sigma} \xi, \alpha)}\right) \\
& +a_{2}\left(\frac{-\sigma B-D \sqrt{-\sigma} \tanh (\sqrt{-\sigma} \xi, \alpha)}{D-B \sqrt{-\sigma} \tanh (\sqrt{-\sigma} \xi, \alpha)}\right)^{2},
\end{aligned}
$$

where $\sigma<0, \xi=x+c t$,

$$
\begin{aligned}
u= & a_{0}+a_{1}\left(\frac{-\sigma B-D \sqrt{-\sigma} \operatorname{coth}(\sqrt{-\sigma} \xi, \alpha)}{D-B \sqrt{-\sigma} \operatorname{coth}(\sqrt{-\sigma} \xi, \alpha)}\right) \\
& +a_{2}\left(\frac{-\sigma B-D \sqrt{-\sigma} \operatorname{coth}(\sqrt{-\sigma} \xi, \alpha)}{D-B \sqrt{-\sigma} \operatorname{coth}(\sqrt{-\sigma} \xi, \alpha)}\right)^{2},
\end{aligned}
$$


where $\sigma<0, \xi=x+c t$,

$$
\begin{aligned}
u= & a_{0}+a_{1}\left(\frac{-\sigma B+D \sqrt{\sigma} \tan (\sqrt{\sigma} \xi, \alpha)}{D+B \sqrt{\sigma} \tan (\sqrt{\sigma} \xi, \alpha)}\right) \\
& +a_{2}\left(\frac{-\sigma B+D \sqrt{\sigma} \tan (\sqrt{\sigma} \xi, \alpha)}{D+B \sqrt{\sigma} \tan (\sqrt{\sigma} \xi, \alpha)}\right)^{2}
\end{aligned}
$$

where $\sigma>0, \xi=x+c t$,

$$
\begin{aligned}
u= & a_{0}+a_{1}\left(\frac{-\sigma B-D \sqrt{\sigma} \cot (\sqrt{\sigma} \xi, \alpha)}{D-B \sqrt{\sigma} \cot (\sqrt{\sigma} \xi, \alpha)}\right) \\
& +a_{2}\left(\frac{-\sigma B-D \sqrt{\sigma} \cot (\sqrt{\sigma} \xi, \alpha)}{D-B \sqrt{\sigma} \cot (\sqrt{\sigma} \xi, \alpha)}\right)^{2}
\end{aligned}
$$

where $\sigma>0, \xi=x+c t$.

If $\sigma=0$, one can get $u=0$.

Using (55), one can get new types of explicit solutions of Eq. (1) as follows:

$$
u=a_{0}+a_{2}\left(\frac{-\sigma B-D \sqrt{-\sigma} \tanh (\sqrt{-\sigma} \xi, \alpha)}{D-B \sqrt{-\sigma} \tanh (\sqrt{-\sigma} \xi, \alpha)}\right)^{2}
$$

where $\sigma<0, \xi=x+c t$,

$$
u=a_{0}+a_{2}\left(\frac{-\sigma B-D \sqrt{-\sigma} \operatorname{coth}(\sqrt{-\sigma} \xi, \alpha)}{D-B \sqrt{-\sigma} \operatorname{coth}(\sqrt{-\sigma} \xi, \alpha)}\right)^{2},
$$

where $\sigma<0, \xi=x+c t$,

$$
u=a_{0}+a_{2}\left(\frac{-\sigma B+D \sqrt{\sigma} \tan (\sqrt{\sigma} \xi, \alpha)}{D+B \sqrt{\sigma} \tan (\sqrt{\sigma} \xi, \alpha)}\right)^{2}
$$

where $\sigma>0, \xi=x+c t$,

$$
u=a_{0}+a_{2}\left(\frac{-\sigma B-D \sqrt{\sigma} \cot (\sqrt{\sigma} \xi, \alpha)}{D-B \sqrt{\sigma} \cot (\sqrt{\sigma} \xi, \alpha)}\right)^{2}
$$

where $\sigma>0, \xi=x+c t$,

$$
u=a_{0}+a_{2}\left(\frac{D \Gamma(1+\alpha)}{-D\left(\xi^{\alpha}+\omega\right)+B \Gamma(1+\alpha)}\right)^{2}
$$

where $\sigma=0, \xi=x+c t$.

Remark 2 Using (53) and (56), we can also get other exact solutions of (1). Here we do not list all of them.

Remark 3 To the best of our knowledge, the solutions obtained in this paper have not been reported in previous literature. Therefore, these solutions are new. 
Remark 4 It is interesting to note that if $\alpha=1$, FDEs (1) can be reduced to the conventional integer order $\mathrm{KdV}$ equation, and the obtained exact solutions can be reduced to the conventional hyperbolic and trigonometric functions.

\section{Concluding remarks}

In the present study, we investigated the symmetry properties, similarity reduction forms and explicit solutions of the time fractional $\mathrm{KdV}$ equation by using the Lie symmetry groups. Lie point symmetry groups of the governing equations were obtained. The obtained point transformation groups, however, for the time fractional $\mathrm{KdV}$ equation are fewer than those for the generalized $\mathrm{KdV}$ equation. The main reason is that the fractional order $0<\alpha \leq 1$ is an arbitrary parameter in our model. Based on the obtained Lie point symmetries, we have shown that this equation can transform into a nonlinear ordinary differential equation of fractional order with the new independent variable $\xi=x t^{-\alpha / 3}$. The derivative is an Erdélyi-Kober derivative depending on a parameter $\alpha$. At last, some exact and explicit solutions of the equations are presented. These solutions include generalized hyperbolic function solutions, generalized trigonometric function solutions and rational function solutions and so on. These solutions may be useful to further investigate the complicated nonlinear physical phenomena.

Competing interests

The authors declare that they have no competing interests.

Authors' contributions

All authors read and approved the final manuscript.

\section{Acknowledgements}

The authors express their sincere thanks to the referees for their careful review of this manuscript and their useful suggestions. This work was completed with the support of the National Natural Science Foundation of China (NNSFC) (Grant No. 11171022)

Received: 8 September 2013 Accepted: 2 October 2013 Published: 08 Nov 2013

\section{References}

1. Olver, PJ: Application of Lie Group to Differential Equation. Springer, New York (1986)

2. Ovsiannikov, LV: Group Analysis of Differential Equations. Academic Press, New York (1982)

3. Lie, S: On integration of a class of linear partial differential equations by means of definite integrals. Arch. Math. Log 6(3), 328-368 (1881)

4. Bluman, GW, Kumei, S: Symmetries and Differential Equations. Springer, New York (1989)

5. Ibragimov, NH (ed.): CRC Handbook of Lie Group Analysis of Differential Equations, vol. 1. CRC Press, Boca Raton (1994)

6. Ibragimov, NH (ed.): CRC Handbook of Lie Group Analysis of Differential Equations, vol. 2. CRC Press, Boca Raton (1995)

7. Ibragimov, NH (ed.): CRC Handbook of Lie Group Analysis of Differential Equations, vol. 3. CRC Press, Boca Raton (1996)

8. Liu, HZ, Geng, YX: Symmetry reductions and exact solutions to the systems of carbon nanotubes conveying fluid. J. Differ. Equ. 254, 2289-2303 (2013)

9. Craddock, M, Lennox, K: Lie symmetry methods for multi-dimensional parabolic PDEs and diffusions. J. Differ. Equ. 252, 56-90 (2012)

10. Kumar, S, Singh, K, Gupta, RK: Painlevé analysis, Lie symmetries and exact solutions for $(2+1)$-dimensional variable coefficients Broer-Kaup equations. Commun. Nonlinear Sci. Numer. Simul. 17, 1529-1541 (2012)

11. Vaneeva, O: Lie symmetries and exact solutions of variable coefficient $\mathrm{mKdV}$ equations: an equivalence based approach. Commun. Nonlinear Sci. Numer. Simul. 17, 611-618 (2012)

12. Naz, R, Khan, MD, Naeem, l: Conservation laws and exact solutions of a class of non linear regularized long wave equations via double reduction theory and Lie symmetries. Commun. Nonlinear Sci. Numer. Simul. 18, 826-834 (2013)

13. Listopadovaa, $V$, Magdab, O, Pobyzhc, V: How to find solutions, Lie symmetries, and conservation laws of forced Korteweg-de Vries equations in optimal way. Nonlinear Anal., Real World Appl. 14, 202-205 (2013)

14. Johnpillaia, AG, Karab, AH, Biswas, A: Symmetry reduction, exact group-invariant solutions and conservation laws of the Benjamin-Bona-Mahoney equation. Appl. Math. Lett. 26, 376-381 (2013)

15. Jefferson, GF: On the second-order approximate symmetry classification and optimal systems of subalgebras for a forced Korteweg-de Vries equation. Commun. Nonlinear Sci. Numer. Simul. 18, 2340-2358 (2013) 
16. Cherniha, R, Pliukhin, O: New conditional symmetries and exact solutions of reaction-diffusion-convection equations with exponential nonlinearities. J. Math. Anal. Appl. 403, 23-37 (2013)

17. Gazizov, RK, Kasatkin, AA, Lukashchuk, YS: Continuous transformation groups of fractional differential equations. Vestn. USATU 9, 125-135 (2007) (in Russian)

18. Gazizov, RK, Kasatkin, AA, Lukashchuk, SY: Symmetry properties of fractional diffusion equations. Phys. Scr. T 136, 014016 (2009)

19. Buckwar, E, Luchko, Y: Invariance of a partial differential equation of fractional order under the Lie group of scaling transformations. J. Math. Anal. Appl. 227, 81-97 (1998)

20. Djordjevic, VD, Atanackovic, TM: Similarity solutions to nonlinear heat conduction and Burgers/Korteweg-de Vries fractional equations. J. Comput. Appl. Math. 212, 701-714 (2008)

21. Sahadevan, R, Bakkyaraj, T: Invariant analysis of time fractional generalized Burgers and Korteweg-de Vries equations. J. Math. Anal. Appl. 393, 341-347 (2012)

22. Wang, GW, Liu, XQ, Zhang, YY: Lie symmetry analysis to the time fractional generalized fifth-order KdV equation. Commun. Nonlinear Sci. Numer. Simul. 18, 2321-2326 (2013)

23. Liu, HZ: Complete group classifications and symmetry reductions of the fractional fifth-order KdV types of equations. Stud. Appl. Math. (2013). doi:10.1111/sapm.12011

24. Diethelm, K: The Analysis of Fractional Differential Equations. Springer, Berlin (2010)

25. Miller, KS, Ross, B: An Introduction to the Fractional Calculus and Fractional Differential Equations. Wiley, New York (1993)

26. Podlubny, I: Fractional Differential Equations. Academic Press, San Diego (1999)

27. Oldham, KB, Spanier, J: The Fractional Calculus. Academic Press, San Diego (1974)

28. Kiryakova, V: Generalised Fractional Calculus and Applications. Pitman Res. Notes in Math., vol. 301 (1994)

29. El-Sayed, AMA, Gaber, M: The Adomian decomposition method for solving partial differential equations of fractal order in finite domains. Phys. Lett. A 359, 175-182 (2006)

30. Chen, Y, An, HL: Numerical solutions of coupled Burgers equations with time- and space-fractional derivatives. Appl. Math. Comput. 200, 87-95 (2008)

31. Gazizov, RK, Kasatkin, AA: Construction of exact solutions for fractional order differential equations by the invariant subspace method. Comput. Math. Appl. (2013). doi:10.1016/j.camwa.2013.05.006

32. Odibat, Z, Momani, S: A generalized differential transform method for linear partial differential equations of fractional order. Appl. Math. Lett. 21, 194-199 (2008)

33. $L i, X, C h e n, W$ : Analytical study on the fractional anomalous diffusion in a half-plane. J. Phys. A, Math. Theor. 43(49), $495206(2010)$

34. He, JH: A coupling method of a homotopy technique and a perturbation technique for non-linear problems. Int. J. Non-Linear Mech. 35, 37-43 (2000)

35. Wu, G, Lee, EWM: Fractional variational iteration method and its application. Phys. Lett. A 374, 2506-2509 (2010)

36. Zhang, S, Zhang, HQ: Fractional sub-equation method and its applications to nonlinear fractional PDEs. Phys. Lett. A 375, 1069-1073 (2011)

37. Guo, S, Mei, LQ, Li, Y, Sun, YF: The improved fractional sub-equation method and its applications to the space-time fractional differential equations in fluid mechanics. Phys. Lett. A 376, 407-411 (2012)

38. Lu, B: Bäklund transformation of fractional Riccati equation and its applications to nonlinear fractional partial differential equations. Phys. Lett. A 376, 2045-2048 (2012)

39. Jumarie, G: Modified Riemann-Liouville derivative and fractional Taylor series of nondifferentiable functions further results. Comput. Math. Appl. 51, 1367-1376 (2006)

40. Jumarie, G: Cauchy's integral formula via the modified Riemann-Liouville derivative for analytic functions of fractional order. Appl. Math. Lett. 23, 1444-1450 (2010)

\section{Submit your manuscript to a SpringerOpen ${ }^{\ominus}$ journal and benefit from:}

- Convenient online submission

- Rigorous peer review

- Immediate publication on acceptance

- Open access: articles freely available online

- High visibility within the field

- Retaining the copyright to your article 